\title{
NONLINEAR STABILITY OF STATIONARY DISCRETE SHOCKS FOR NONCONVEX SCALAR CONSERVATION LAWS
}

\author{
HAILIANG LIU AND JINGHUA WANG
}

\begin{abstract}
This paper is to study the asymptotic stability of stationary discrete shocks for the Lax-Friedrichs scheme approximating nonconvex scalar conservation laws, provided that the summations of the initial perturbations equal to zero. The result is proved by using a weighted energy method based on the nonconvexity. Moreover, the $l^{1}$ stability is also obtained. The key points of our proofs are to choose a suitable weight function.
\end{abstract}

\section{INTRODUCTION}

We investigate the asymptotic stability of the numerical approximation of the following Riemann problem for nonconvex scalar conservation laws:

$$
u_{t}+f(u)_{x}=0, \quad u(x, 0)=u_{0}(x)= \begin{cases}u_{-}, & x<0 \\ u_{+}, & x>0 .\end{cases}
$$

The corresponding shock wave solution is

$$
u(t, x)= \begin{cases}u_{-}, & x-s t<0 \\ u_{+}, & x-s t>0\end{cases}
$$

where the end states $u_{ \pm}$and related shock speed $s$ satisfy the Rankine-Hugoniot condition

$$
-s\left(u_{+}-u_{-}\right)+f\left(u_{+}\right)-f\left(u_{-}\right)=0
$$

and Oleinik's shock condition

$$
\left(u_{+}-u_{-}\right) Q \equiv\left(u_{+}-u_{-}\right)\left[f(u)-f\left(u_{ \pm}\right)-s\left(u-u_{ \pm}\right)\right]>0
$$

for $u \in\left(\min \left(u_{-}, u_{+}\right), \max \left(u_{-}, u_{+}\right)\right)$. It is noted that when $s \neq f^{\prime}\left(u_{ \pm}\right)$, then (1.3b) implies the Lax shock condition

$$
f^{\prime}\left(u_{+}\right)<s<f^{\prime}\left(u_{-}\right) .
$$

We approximate (1.1) by the Lax-Friedrichs (LF) scheme

$$
u_{j}^{n+1}-u_{j}^{n}+\frac{\lambda}{2}\left(f\left(u_{j+1}^{n}\right)-f\left(u_{j-1}^{n}\right)\right)=\frac{\alpha}{2}\left(u_{j+1}^{n}-2 u_{j}^{n}+u_{j-1}^{n}\right),
$$

Received by the editor January 11, 1995.

1991 Mathematics Subject Classification. Primary 39A11; Secondary 35L65.

Key words and phrases. Stability, discrete shocks, Lax-Friedrichs scheme.

The first author was supported in part by the Science Foundation of Education of Commission of Henan Province and by the Institute of Mathematics, Academia Sinica.

The second author was supported in part by the National Natural Science Foundation of China and by The Raymond and Beverly Sackler Institute of Scientific Computation.

(C)1996 American Mathematical Society 
where $u_{j}^{n}$ is an approximation of $u\left(x_{j}, t_{n}\right), x_{j}=j \Delta x$ and $t_{n}=n \Delta t$, with $\Delta x$ and $\Delta t$ being the spatial and the temporal grid sizes; $\alpha$ is a constant satisfying $0<\alpha<1$, and the temporal and spatial grid ratio $\lambda=\frac{\Delta t}{\Delta x}$ satisfies the CourantFriedrichs-Levy (CFL) condition,

$$
\lambda \max \left|f^{\prime}\right| \leq \alpha .
$$

Corresponding to the difference equation (1.4) we have the following viscous conservation law,

$$
u_{t}+f(u)_{x}=\mu u_{x x}, \quad \mu>0,
$$

which has a shock profile solution $u=U(x-s t)$ satisfying

$$
U(z) \rightarrow u_{ \pm} \text {as } z \rightarrow \pm \infty .
$$

For convenience, we assume that $u_{+}<u_{-}$and $s \neq f^{\prime}\left(u_{ \pm}\right)$; then $U^{\prime}(z)<0$ and $U-u_{ \pm} \sim O(1) \exp (-c|z|)$ as $z \rightarrow \pm \infty$. Hence, the shock profile of (1.6) has the following property:

$$
u(x, t+\Delta t)=u(x-s \Delta t, t) .
$$

Since the solutions of difference equations are only defined on the grid nodes, (1.7) does not always make sense. The standard method to overcome this difficulty is to construct a refined grid

$$
L_{\eta}=\{m \eta+n \mid \eta=s \lambda, \quad m, n \in \mathbf{Z}\},
$$

or to use the expanded grid with $\Delta t_{q}=q \Delta t$, where $\eta=\frac{p}{q}$ is rational with $p$ and $q$ relatively prime (see [29]).

From now on we focus on the discrete shock profile solution $\phi_{j}$ of (1.4), i.e.,

$$
\begin{gathered}
\lambda\left(f\left(\phi_{j+1}\right)-f\left(\phi_{j-1}\right)\right)=\alpha\left(\phi_{j+1}-2 \phi_{j}+\phi_{j-1}\right), \\
\phi_{j} \rightarrow u_{ \pm} \quad \text { as } j \rightarrow \pm \infty,
\end{gathered}
$$

which is called a stationary discrete shock. Its existence and properties have been proved by Jennings [9] provided $\left(u_{-}, u_{+}\right)$satisfies $(1.3 \mathrm{a})-(1.3 \mathrm{~b})$.

The main theorems are the following $l^{2}$ and $l^{\infty}$ asymptotic stability and $l^{1}$ boundedness for the discrete shock wave for the L-F scheme (1.4). We have

Theorem 1.1. Suppose that (1.3a)-(1.3c) and (1.5) (CFL condition) hold. Let $\phi_{j}$ be the stationary discrete shock profile (1.8a) connecting $u_{+}$to $u_{-}$. We assume

$$
\sum_{j}\left(u_{j}^{0}-\phi_{j}^{0}\right)=0
$$

and

$$
\sum_{j=-\infty}^{\infty}\left(1+j^{2}\right)^{\beta}\left|u_{j}^{0}-\phi_{j}\right|^{2} \leq c_{1} \quad(\beta>1)
$$

for some (suitably small) positive constant $c_{1}$. Then there exists a unique global solution, $u_{j}^{n}$, to the L-F scheme (1.4) with initial data $u_{j}^{0}$, and it satisfies

$$
\sup _{j}\left|u_{j}^{n}-\phi_{j}\right| \rightarrow 0 \quad \text { as } n \rightarrow \infty .
$$


Theorem 1.2. Under the hypothesis of Theorem 1.1 and the additional assumption

$$
\sum_{j=-\infty}^{\infty}\left(1+j^{2}\right)^{3 / 2}\left|u_{j}^{0}-\phi_{j}\right|^{2}<\infty
$$

we have

$$
\sup _{0 \leq n<\infty} \sum\left|u_{j}^{n}-\phi_{j}\right|<\infty .
$$

Corollary 1.1. Under the hypothesis of Theorem 1.1 we have $l^{p}(p>1)$ asymptotic stability,

$$
\lim _{n \rightarrow \infty} \sum_{j}\left|u_{j}^{n}-\phi_{j}\right|^{p}=0 .
$$

Remark 1.1. In the original L-F scheme [14], one has $\alpha=1$. However, we do not expect asymptotic stability of the discrete shock profiles in this case. In fact, Liu and Xin [19] verified that stationary discrete shock profiles of the L-F scheme for the scalar equations are not asymptotically stable. Recently, L.A.Ying and T. Zhou [29] proved, based on the energy integration method, that the solution on the odd grid nodes and on the even grid nodes tends to two discrete shocks, respectively. It is interesting to discuss the original L-F scheme for nonconvex $f$.

Our stability analysis is strongly motivated by the nonlinear stability of the viscous shock profile for viscous conservation laws of the form (1.6). There have been extensive studies in the last three decades $[7,11,17,26]$. Recently, some important papers on developing the stability theory for nonconvex equations (see $[12,16,10,24])$ appeared. They showed that a viscous shock profile is nonlinearly stable in the sense that a small initial disturbance, under suitable restrictions, will die out as time tends to infinity. The methods use the weighted energy method, spectral analysis, and semigroups.

The study of existence and stability of discrete shocks is important in understanding the convergence behavior of numerical shock computations. Jennings [9] first investigated the existence and stability of discrete shocks for scalar difference equations. But the work is restricted to strictly monotone schemes; that is, if we denote the scheme as

$$
u_{j}^{n+1}=G\left(u_{j-r}^{n}, u_{j-r+1}^{n}, \cdots, u_{j+t}^{n}\right),
$$

then the first-order derivatives of $G$ with respect to its arguments must be positive. Engquist and Osher proved the stability of first-order monotone schemes in the scalar case [6]. Smyrlis [25] proved stability of a scalar stationary discrete shock wave for the Lax-Wendroff scheme. For scalar conservation laws, the L-F scheme belongs to the class of monotone schemes, which have been well studied, see [13, 23], etc. It has been shown by Kuznecov [13] that the best rate of convergence in the $L^{1}$-norm for such schemes with general BV initial data is of order $(\Delta x)^{1 / 2}$. Teng and Zhang [28] recently derived optimal $L^{1}$-error bounds of $O(\Delta x)$ for monotone difference schemes to an initial value problem for nonconvex scalar conservation laws with initial data being a finite number of piecewise constants, subject to the initial discontinuities satisfying the entropy conditions. Tadmor [27] studied the large-time behavior for the rarefaction waves for some monotone schemes. See also [8]. 
In the case that the far field is a constant state, Chern [2] proved stability of the L-F scheme using diffusion waves. Liu and Xin [18] proved that, for the L-F scheme, the solutions of Riemann problems are single or multiple shocks; and if the summation of the initial perturbations is equal to zero, then the scheme solutions are asymptotically stable. They also study the stability of stationary discrete shocks in [19]. The existence of discrete shock waves of first-order accurate finite difference methods for systems of conservation laws was established by Majda and Ralston [20] by means of the center manifold theorem.

The L-F scheme has been playing important roles both in the theory and numerical computations of hyperbolic conservation laws. In the 1950s, Oleinik [22] studied the existence of global solutions for single conservation laws by this scheme. In the 1980s, DiPerna [5] and X.X. Ding, G.Q. Chen and P.Z. Luo [3, 4, 1] also used it to prove the existence of weak solutions with large amplitude for some $2 \times 2$ systems. The L-F scheme also played an important role in the development of difference methods. It is a representative for monotone schemes. For monotone schemes, there have been systematic theories (see [9, 15, 21]).

In this paper we develop the stability theory of discrete shocks for the L-F scheme in the case of nonconvex nonlinearities. In $\S 2$, we first state some results on the existence and properties of discrete shocks. Then we study the nonlinear stability of discrete shocks. We use a weighted energy method. Owing to the nonconvexity of $f$ and the discrete errors, our analysis is technically rather involved. Based on the same idea, the stability problem of its continuous counterpart has also been solved recently (see [16]).

In $\S 3$, we investigate $l^{1}$ stability of discrete shocks. In contrast with the convex case, because of the nonmonotonicity of $f^{\prime}$, even the linear stability analysis in the $l^{1}$-norm is difficult. We overcome this difficulty by carefully choosing weights. The solution can be estimated by using the essential monotonicity of the scheme. This, together with the $l^{2}$-stability analysis, yields the desired result in Theorem 1.2.

\section{The $l^{2}$ STABILITY ANALYSiS}

In this section we proceed to prove Theorem 1.1, the nonlinear stability of stationary discrete shocks.

First we notice the fact that a shock profile of (1.4) depends continuously on its value at a point (see [9]). From this, the following two lemmas follow; we omit proofs.

Lemma 2.1. Assume (1.3a)-(1.3b) and $u_{+}<u_{-}$for $s=0$. Then there exists a stationary discrete shock profile to (1.4), i.e., (1.8a)-(1.8b) holds. Furthermore, the $\phi_{j}$ satisfy

$$
\phi_{j}>\phi_{j+1} \quad \text { for } j \in Z \text {. }
$$

Lemma 2.2. Let $u_{j}^{0}(j \in Z)$ be initial values satisfying

$$
\sum_{j \in Z}\left|u_{j}^{0}-\psi_{j}\right| \leq \delta
$$

where $\psi_{j}$ is a stationary discrete shock profile of the scheme (1.4). Then there must exist another shock profile $\phi_{j}$ such that

$$
\sum_{j \in Z}\left(u_{j}^{0}-\phi_{j}\right)=0
$$


and

$$
\sum_{j \in Z}\left|u_{j}^{0}-\phi_{j}\right| \leq 2 \delta
$$

To prove the stability result, we reformulate the problem as follows. Let $u_{j}^{n}$ be a solution of the L-F scheme (1.4) with initial data $u_{j}^{0}$. From Lemma 2.2, if $\sum_{j}\left(u_{j}^{0}-\psi_{j}\right) \neq 0$, we still can find a discrete shock $\phi_{j}$ of the scheme (1.8a) such that

$$
\sum_{j \in Z}\left(u_{j}^{0}-\phi_{j}\right)=0
$$

thus, the assumption on the initial perturbation, (1.9), does not restrict generality.

Setting

$$
v_{j}^{n}=\sum_{k=-\infty}^{j}\left(u_{k}^{n}-\phi_{k}\right),
$$

we have $v_{j}^{n} \rightarrow 0$ as $j \rightarrow \pm \infty$. Subtracting (1.8a) from (1.4), and summing up the resulting expression from $-\infty$ to $j$, we get

$$
\begin{aligned}
v_{j}^{n+1} & -v_{j}^{n}+\frac{\lambda}{2}\left(f\left(u_{j+1}^{n}\right)-f\left(\phi_{j+1}\right)\right)+\frac{\lambda}{2}\left(f\left(u_{j}^{n}\right)-f\left(\phi_{j}\right)\right) \\
& =\frac{\alpha}{2}\left(v_{j+1}^{n}-2 v_{j}^{n}+v_{j-1}^{n}\right) .
\end{aligned}
$$

Noting that $u_{j}^{n}-\phi_{j}=v_{j}^{n}-v_{j-1}^{n}$, we have

$$
\begin{aligned}
v_{j}^{n+1}- & v_{j}^{n}+\frac{\lambda}{2} f^{\prime}\left(\phi_{j+1}^{n}\right)\left(v_{j+1}^{n}-v_{j}^{n}\right)+\frac{\lambda}{2} f^{\prime}\left(\phi_{j}^{n}\right)\left(v_{j}^{n}-v_{j-1}^{n}\right) \\
& +\frac{\lambda}{2} F\left(\phi_{j+1}^{n}, v_{j+1}^{n}-v_{j}^{n}\right)+\frac{\lambda}{2} F\left(\phi_{j}^{n}, v_{j}^{n}-v_{j-1}^{n}\right)=\frac{\alpha}{2}\left(v_{j+1}^{n}-2 v_{j}^{n}+v_{j-1}^{n}\right),
\end{aligned}
$$

where

$$
F(\phi, u-\phi)=f(u)-f(\phi)-f^{\prime}(\phi)(u-\phi)
$$

satisfies the estimate

$$
|F(\phi, u-\phi)| \leq O(1)|u-\phi|^{2},
$$

and $O(1)$ is a positive constant. Using the notations

$$
\Lambda_{j}=f^{\prime}\left(\phi_{j}\right), \quad \theta_{j}^{n}=F\left(\phi_{j}, v_{j}^{n}-v_{j-1}^{n}\right),
$$

we may rewrite the equation (2.4) as

$$
\begin{gathered}
v_{j}^{n+1}-v_{j}^{n}+\frac{\lambda}{2} \Lambda_{j+1}\left(v_{j+1}^{n}-v_{j}^{n}\right)+\frac{\lambda}{2} \Lambda_{j}\left(v_{j}^{n}-v_{j-1}^{n}\right) \\
-\frac{\alpha}{2}\left(v_{j+1}^{n}-2 v_{j}^{n}+v_{j-1}^{n}\right)=e_{j}^{n},
\end{gathered}
$$

where

$$
e_{j}^{n}=-\frac{\lambda}{2}\left(\theta_{j+1}^{n}+\theta_{j}^{n}\right)
$$


Before we derive our basic energy estimate, we first explain why the standard energy method does not work in our problem with nonconvex flux $f$. We take the scalar product of equation (2.4) with $2 v_{j}^{n}$, and using summation by parts, we obtain

$$
\begin{aligned}
& \sum_{j}\left(v_{j}^{n+1}\right)^{2}-\sum_{j}\left(v_{j}^{n}\right)^{2}+\alpha \sum_{j}\left(v_{j+1}^{n}-v_{j}^{n}\right)^{2}+\lambda \sum_{j}\left(\Lambda_{j}-\Lambda_{j+1}\right)\left(v_{j}^{n}\right)^{2} \\
& =\sum_{j}\left(v_{j+1}^{n+1}-v_{j}^{n}\right)^{2}+2 \sum_{j} v_{j}^{n} e_{j}^{n},
\end{aligned}
$$

where we have used the identity

$$
\sum_{j} v_{j}^{n}\left(2 v_{j}^{n}-v_{j+1}^{n}-v_{j-1}^{n}\right)=\sum_{j}\left(v_{j+1}^{n}-v_{j}^{n}\right)^{2} .
$$

From (2.7) we see that $\Lambda_{j}-\Lambda_{j+1}$ in general changes its sign because of the nonconvexity of $f$, and hence the summation of (2.7) does not give any useful estimate. That is, the standard energy integration method based on (2.7) does not work in our problem.

In view of the above consideration, to derive an a priori basic $l^{2}$ estimate of (2.1), we introduce a weight function $W(u)>0$ belonging to $C^{2}\left[u_{+}, u_{-}\right]$. Setting $W_{j}=$ $W\left(\phi_{j}\right)$, then multiplying (2.6) by $2 v_{j}^{n} W_{j}$ and summing over $j$, we obtain

$$
\begin{gathered}
\sum_{j} 2\left(v_{j}^{n+1}-v_{j}^{n}\right) v_{j}^{n} W_{j}+\lambda\left\{\sum_{j} \Lambda_{j+1} v_{j}^{n} W_{j}\left(v_{j+1}^{n}-v_{j}^{n}\right)+\sum_{j} \Lambda_{j} v_{j}^{n} W_{j}\left(v_{j}^{n}-v_{j-1}^{n}\right)\right\} \\
+\alpha \sum_{j} v_{j}^{n} W_{j}\left(2 v_{j}^{n}-v_{j+1}^{n}-v_{j-1}^{n}\right)=2 \sum_{j} v_{j}^{n} W_{j} e_{j}^{n} .
\end{gathered}
$$

We now successively estimate each term $I_{i} \quad(i=1,2,3)$ on the left-hand side of (2.8), denoting

$$
\left|v_{j}^{n}\right|_{w}^{2}=\left(v_{j}^{n}\right)^{2} W_{j}
$$

We have

$$
\begin{aligned}
I_{1}= & \sum_{j}\left\{\left(v_{j}^{n+1}\right)^{2}-\left(v_{j}^{n+1}-v_{j}^{n}\right)^{2}-\left(v_{j}^{n}\right)^{2}\right\} W_{j} \\
= & \sum_{j}\left|v_{j}^{n+1}\right|_{w}^{2}-\sum_{j}\left|v_{j}^{n}\right|_{w}^{2}-\sum_{j}\left|v_{j}^{n+1}-v_{j}^{n}\right|_{w}^{2}, \\
I_{2}= & \lambda\left\{\sum_{j} \Lambda_{j+1} W_{j} v_{j}^{n} v_{j+1}^{n}-\sum_{j} \Lambda_{j+1} W_{j}\left(v_{j}^{n}\right)^{2}\right. \\
& \left.+\sum_{j} \Lambda_{j} W_{j}\left(v_{j}^{n}\right)^{2}-\sum_{j} \Lambda_{j} W_{j} v_{j}^{n} v_{j-1}^{n}\right\} \\
= & \lambda\left\{-\sum_{j}\left(\Lambda_{j+1} W_{j+1}-\Lambda_{j} W_{j}\right)\left(v_{j}^{n}\right)^{2}\right. \\
& \left.+\sum_{j} \Lambda_{j+1}\left(W_{j}-W_{j+1}\right) v_{j}^{n}\left(v_{j+1}^{n}-v_{j}^{n}\right)\right\},
\end{aligned}
$$




$$
\begin{aligned}
I_{3}= & \alpha\left\{\sum_{j} v_{j}^{n} W_{j}\left(v_{j}^{n}-v_{j+1}^{n}\right)-\sum_{j} v_{j}^{n} W_{j}\left(v_{j-1}^{n}-v_{j}^{n}\right)\right\} \\
= & \alpha\left\{\sum_{j} \frac{W_{j}}{2}\left[\left(v_{j}^{n}-v_{j+1}^{n}\right)^{2}+\left(v_{j}^{n}\right)^{2}-\left(v_{j+1}^{n}\right)^{2}\right]\right. \\
& \left.+\sum_{j} \frac{W_{j+1}}{2}\left[\left(v_{j}^{n}-v_{j+1}\right)^{2}+\left(v_{j+1}^{n}\right)^{2}-\left(v_{j}^{n}\right)^{2}\right]\right\} \\
= & \alpha\left\{\sum_{j}\left(v_{j}^{n}-v_{j+1}^{n}\right)^{2} \frac{W_{j}+W_{j+1}}{2}+\sum_{j}\left(v_{j}^{n}\right)^{2}\left(W_{j}-\frac{W_{j+1}+W_{j-1}}{2}\right)\right\} .
\end{aligned}
$$

Then

$$
\begin{gathered}
\sum_{j}\left|v_{j}^{n+1}\right|_{w}^{2}-\sum_{j}\left|v_{j}^{n}\right|_{w}^{2}+\sum_{j} A_{j}\left(v_{j}^{n}\right)^{2}+\alpha \sum_{j} \frac{W_{j}+W_{j+1}}{2}\left|v_{j+1}^{n}-v_{j}^{n}\right|^{2} \\
=\sum_{j}\left|v_{j}^{n+1}-v_{j}^{n}\right|_{w}^{2}-\sum_{j} B_{j}^{n}+2 \sum_{j} v_{j}^{n} W_{j} e_{j}^{n},
\end{gathered}
$$

where

$$
\begin{aligned}
A_{j} & =\alpha\left(W_{j}-\frac{W_{j+1}+W_{j-1}}{2}\right)-\lambda\left(\Lambda_{j+1} W_{j+1}-\Lambda_{j} W_{j}\right), \\
B_{j}^{n} & =\lambda v_{j}^{n}\left(W_{j}-W_{j+1}\right) \Lambda_{j+1}\left(v_{j+1}^{n}-v_{j}^{n}\right) .
\end{aligned}
$$

Next we estimate the terms on the right-hand side of (2.9). We set

$$
N\left(n_{1}\right)=\sup _{n \leq n_{1}}\left(\sum_{j}\left|v_{j}^{n}\right|^{2}\right)^{1 / 2}
$$

and assume that $N\left(n_{1}\right)$ is small. Obviously, we have

$$
\sup _{n, j}\left|v_{j}^{n}\right| \leq N\left(n_{1}\right) \text {. }
$$

It follows from equation (2.6) that

$$
\begin{gathered}
v_{j}^{n+1}-v_{j}^{n}=\left\{\frac{\alpha}{2}-\frac{\lambda}{2} \Lambda_{j+1}\right\}\left(v_{j+1}^{n}-v_{j}^{n}\right) \\
-\left\{\frac{\alpha}{2}+\frac{\lambda}{2} \Lambda_{j}\right\}\left(v_{j}^{n}-v_{j-1}^{n}\right)+e_{j}^{n} .
\end{gathered}
$$

Then we have

$$
\begin{aligned}
\left|v_{j}^{n+1}-v_{j}^{n}\right|^{2} \leq & \frac{1}{2}\left\{\left(\alpha-\lambda \Lambda_{j+1}\right)^{2}\left|v_{j+1}^{n}-v_{j}^{n}\right|^{2}+\left(\alpha+\lambda \Lambda_{j}\right)^{2}\left|v_{j}^{n}-v_{j-1}^{n}\right|^{2}\right\} \\
& +O(1)\left\{\left|v_{j+1}^{n}-v_{j}^{n}\right|^{4}+\left|v_{j}^{n}-v_{j-1}^{n}\right|^{4}\right\}
\end{aligned}
$$

where we have used (2.5b). Consequently,

$$
\sum_{j}\left|v_{j}^{n+1}-v_{j}^{n}\right|_{w}^{2} \leq\left(\left(\alpha+\lambda \max \left|f^{\prime}\right|\right)^{2}+O(1) N\left(n_{1}\right)\right) \times \sum_{j}\left|v_{j+1}^{n}-v_{j}^{n}\right|_{w}^{2} .
$$

Next, using (2.5b) and (2.12), we get

$$
2 \sum_{j}\left|v_{j}^{n} W_{j} e_{j}^{n}\right| \leq C N\left(n_{1}\right) \sum_{j}\left|v_{j+1}^{n}-v_{j}^{n}\right|_{w}^{2} .
$$


By the Schwarz inequality we can estimate $B_{j}^{n}$ as follows:

$$
\begin{aligned}
\left|B_{j}^{n}\right| & \leq \sqrt{W_{j}+W_{j+1}}\left|v_{j+1}^{n}-v_{j}^{n}\right| \lambda \sqrt{\left|W_{j+1}-W_{j}\right|}\left|\Lambda_{j+1} v_{j}^{n}\right| \\
& \leq \varepsilon\left(W_{j}+W_{j+1}\right)\left(v_{j+1}^{n}-v_{j}^{n}\right)^{2}+\frac{\left(\lambda \Lambda_{j+1}\right)^{2}}{4 \varepsilon}\left|W_{j+1}-W_{j}\right|\left(v_{j}^{n}\right)^{2} \\
& =\varepsilon\left(W_{j}+W_{j+1}\right)\left(v_{j+1}^{n}-v_{j}^{n}\right)^{2}+\frac{\lambda^{2} \Lambda_{j+1}^{2}}{4 \varepsilon}\left|W^{\prime}(\tilde{\phi})\right|\left|\phi_{j+1}-\phi_{j}\right|\left(v_{j}^{n}\right)^{2} \\
& \leq \varepsilon\left(W_{j}+W_{j+1}\right)\left(v_{j+1}-v_{j}^{n}\right)^{2}+\frac{c_{0} \lambda^{2}}{4 \varepsilon}\left|\phi_{j+1}-\phi_{j}\right|\left(v_{j}^{n}\right)^{2},
\end{aligned}
$$

where $\tilde{\phi}$ is a mean value. We choose the weights

$$
W_{j}=\frac{\left(\phi_{j}-u_{+}\right)\left(\phi_{j}-u_{-}\right)}{Q\left(\phi_{j}\right)},
$$

where

$$
Q(u)=f(u)-f\left(u_{ \pm}\right) .
$$

The specific choice of weights in (2.17) is made to obtain a useful $l^{2}$ a priori estimate. Regarding $A_{j}$, we have the following lemma.

Lemma 2.3. Let $A_{j}$ be defined in (2.10) and $W_{j}$ be the weight defined in (2.17). Then there exists a positive $\nu$ such that

$$
A_{j} \geq \nu \lambda\left(\phi_{j}-\phi_{j+1}\right),
$$

provided $\lambda$ is suitably small.

We defer the proof of this lemma to the end of this section. Assuming Lemma 2.3, we obtain the following basic a priori estimate.

Proposition 2.1. [A priori estimate] Let $v_{j}^{n}$ be a solution of (2.6) for $n \leq n_{1}$. Then there exists a positive constant $C$ independent of $n_{1}$ such that for all $n \leq n_{1}$

$$
\begin{aligned}
\sum_{j}\left|v_{j}^{n}\right|^{2} & +\sum_{n \leq n_{1}} \sum_{j}\left|v_{j+1}^{n}-v_{j}^{n}\right|^{2} \\
& +\sum_{n \leq n_{1}} \sum_{j} \lambda\left(\phi_{j}-\phi_{j+1}\right)\left(v_{j}^{n}\right)^{2} \leq C \sum_{j}\left|v_{j}^{0}\right|^{2},
\end{aligned}
$$

provided $\lambda$ and $N\left(n_{1}\right)$ are suitably small.

Proof. By Lemma 2.3, we collect (2.9), (2.14), (2.15) and (2.16) to obtain

$$
\begin{gathered}
\sum_{j}\left|v_{j}^{n+1}\right|_{w}^{2}-\sum_{j}\left|v_{j}^{n}\right|_{w}^{2}+(\alpha-2 \varepsilon) \sum_{j} \frac{W_{j}+W_{j+1}}{2}\left|v_{j+1}^{n}-v_{j}^{n}\right|^{2} \\
-\left[\left(\alpha+\lambda \max \left|f^{\prime}\right|\right)^{2}+O(1) N\left(n_{1}\right)\right] \sum_{j}\left|v_{j+1}^{n}-v_{j}^{n}\right|_{w}^{2} \\
+\sum_{j}\left(A_{j}-\frac{c_{0} \lambda^{2}}{4 \varepsilon}\left(\phi_{j}-\phi_{j+1}\right)\right)\left|v_{j}^{n}\right|^{2} \leq 0 .
\end{gathered}
$$

Now, we recall the equation (1.8a) for $\phi_{j}$,

$$
\lambda\left(f\left(\phi_{j+1}\right)-f\left(\phi_{j-1}\right)\right)=\alpha\left(\phi_{j+1}-2 \phi_{j}+\phi_{j-1}\right),
$$


and sum it over $j$ from $-\infty$ to $j$ to obtain

$$
\lambda\left(f\left(\phi_{j+1}\right)-2 f\left(u_{ \pm}\right)+f\left(\phi_{j}\right)\right)=\alpha\left(\phi_{j+1}-\phi_{j}\right),
$$

that is,

$$
\alpha\left(\phi_{j+1}-\phi_{j}\right)=\lambda\left(Q_{j+1}+Q_{j}\right)
$$

By the expression of $W$ and the fact $f^{\prime}\left(u_{ \pm}\right) \neq 0$, there exists a positive constant $C$ such that

$$
C^{-1} \leq W_{j} \leq C
$$

Combining (2.21) and (2.22), we have

$$
\begin{aligned}
\left|\frac{W_{j+1}-W_{j}}{2 W_{j}}\right| & =\left|\frac{W^{\prime}\left(\bar{\phi}_{j}\right)}{2 W_{j}}\left(\phi_{j+1}-\phi_{j}\right)\right| \\
& =\lambda\left|\frac{W^{\prime}\left(\bar{\phi}_{j}\right)}{2 \alpha W_{j}}\left(Q_{j+1}+Q_{j}\right)\right| \\
& \leq C \lambda,
\end{aligned}
$$

where $\bar{\phi}_{j}$ is a mean value and $C$ is a positive constant. Thus we obtain

$$
\begin{aligned}
\sum_{j} \frac{W_{j}+W_{j+1}}{2}\left|v_{j+1}^{n}-v_{j}^{n}\right|^{2} & =\sum_{j}\left|v_{j+1}^{n}-v_{j}^{n}\right|^{2} W_{j}+\sum_{j} \frac{W_{j+1}-W_{j}}{2 W_{j}}\left|v_{j+1}^{n}-v_{j}^{n}\right|^{2} W_{j} \\
& \geq(1-C \lambda) \sum_{j}\left|v_{j+1}^{n}-v_{j}^{n}\right|_{w}^{2} .
\end{aligned}
$$

Substituting (2.23) into (2.20) yields

$$
\begin{aligned}
& \sum_{j}\left|v_{j}^{n+1}\right|_{w}^{2}-\sum_{j}\left|v_{j}^{n}\right|_{w}^{2}+\sum_{j}\left(A_{j}-\frac{c_{0} \lambda^{2}}{4 \varepsilon}\left(\phi_{j}-\phi_{j+1}\right)\right)\left|v_{j}^{n}\right|^{2} \\
& \quad+\left(\alpha-\left(\alpha+\lambda \max \left|f^{\prime}\right|\right)^{2}-2 \varepsilon-O(1) N\left(n_{1}\right)-O(1) \lambda\right) \sum_{j}\left|v_{j+1}^{n}-v_{j}^{n}\right|_{w}^{2} \leq 0 .
\end{aligned}
$$

Using (2.18), we have

$$
A_{j}-\frac{c_{0} \lambda^{2}}{4 \varepsilon}\left(\phi_{j}-\phi_{j+1}\right) \geq \lambda\left(\nu-\frac{c_{0} \lambda}{4 \varepsilon}\right)\left(\phi_{j}-\phi_{j+1}\right) .
$$

Since $\alpha<1$, we can take two suitably small positive constants $\varepsilon_{0}$ and $\lambda_{0}$ such that if $\varepsilon<\varepsilon_{0}$ and $\lambda<\lambda_{0}$ we have

$$
\alpha-\left(\alpha+\lambda \max \left|f^{\prime}\right|\right)^{2}-2 \varepsilon-O(1) N\left(n_{1}\right)-O(1) \lambda>0
$$

provided $N\left(n_{1}\right)$ is suitably small $\left(N\left(n_{1}\right)<\bar{\delta}\right)$. Then for this fixed $\varepsilon_{0}$, choosing $\lambda$ to satisfy $\lambda<\min \left(\lambda_{0}, \frac{4 \nu \varepsilon_{0}}{c_{0}}\right)$, we have

$$
\nu-\frac{c_{0} \lambda}{4 \varepsilon_{0}}>0
$$


Finally, summing the two sides of (2.24) from 0 to $n_{1}$ with respect to $n$, by virtue of (2.22), (2.25)- (2.27), we have

$$
\begin{aligned}
\sum_{j}\left|v_{j}^{n+1}\right|^{2} & +\sum_{n \leq n_{1}} \sum_{j}\left|v_{j+1}^{n}-v_{j}^{n}\right|^{2} \\
& +\sum_{n \leq n_{1}} \sum_{j} \lambda\left(\phi_{j}-\phi_{j+1}\right)\left(v_{j}^{n}\right)^{2} \leq C \sum_{j}\left|v_{j}^{0}\right|^{2}
\end{aligned}
$$

and Proposition 2.1 immediately follows.

By (2.6), $v_{j}^{n+1}$ can be expressed in terms of $v_{j}^{n}$ in the explicit scheme; we can thus obtain $v_{j}^{n}$ step by step from the beginning at $n=0$. Moreover, we can estimate the $l^{2}$-norm of $v_{j}^{n}$ as follows:

$$
\sum_{j}\left(v_{j}^{n+1}\right)^{2} \leq C \sum_{j}\left(v_{j}^{n}\right)^{2} .
$$

Combining (2.29) with Proposition 2.1 and a standard continuity argument, we see that the following proposition holds.

Proposition 2.2. Assume that $N(0)$ is suitably small. Then the problem (2.6) has a unique global solution $v_{j}^{n}$ satisfying, for any $n \geq 0$,

$$
\sup _{n} \sum_{j}\left|v_{j}^{n}\right|^{2}+\sum_{j, n}\left|v_{j}^{n}-v_{j+1}^{n}\right|^{2}+\sum_{j, n}\left(\phi_{j}-\phi_{j+1}\right)\left|v_{j}^{n}\right|^{2} \leq C N^{2}(0)
$$

where $C$ is a positive constant independent of $n$ and $j$.

We now turn to prove our main Theorem 1.1.

Proof. First we prove that the conditions (1.10) on the initial data imply that $N(0)$ is small. Here we give a proof under the condition

$$
\sum_{j=-\infty}^{\infty}\left(1+j^{2}\right)^{\beta}\left|u_{j}^{0}-\phi_{j}\right|^{2} \leq c_{1}
$$

for any given constant $\beta>1$ and $c_{1}$ a suitably small constant. Applying the Hölder inequality to

$$
v_{j}^{0}=\sum_{k=-\infty}^{j}\left(u_{k}^{0}-\phi_{k}\right),
$$

we have

$$
\begin{aligned}
\left|v_{j}^{0}\right|^{2} & \leq \sum_{k=-\infty}^{j}\left(1+k^{2}\right)^{\beta}\left|u_{k}^{0}-\phi_{k}\right|^{2} \sum_{k=-\infty}^{j}\left(1+k^{2}\right)^{-\beta} \\
& \leq c_{1} \sum_{k=-\infty}^{j}\left(1+k^{2}\right)^{-\beta}
\end{aligned}
$$


Therefore,

$$
\begin{aligned}
\sum_{j}\left|v_{j}^{0}\right|^{2} & \leq c_{1} \sum_{j} \sum_{k=-\infty}^{j}\left(1+k^{2}\right)^{-\beta} \\
& \leq c_{1} \int_{-\infty}^{+\infty} \int_{-\infty}^{x}\left(1+y^{2}\right)^{-\beta} d y d x \\
& \leq \frac{c_{1}}{\beta-1} O(1),
\end{aligned}
$$

So

$$
N(0)=\left(\sum_{j}\left|v_{j}^{0}\right|^{2}\right)^{1 / 2} \leq O(1) \sqrt{c_{1}} .
$$

Thus, the hypothesis in Proposition 2.2 is fulfilled under the condition (1.10). It follows from Proposition 2.2 that there exists a unique global solution, $u_{j}^{n}$, to the $\mathrm{L}-\mathrm{F}$ scheme (1.4) in view of the relation

$$
u_{j}^{n}=\phi_{j}+v_{j}^{n}-v_{j-1}^{n},
$$

which follows from (2.2). Next we study the asymptotic behavior of the solution $u_{j}^{n}$ to (1.4). It follows from Proposition 2.2 that

$$
\sum_{n=1}^{\infty}\left(\sum_{j}\left|v_{j}^{n}-v_{j+1}^{n}\right|^{2}\right)<+\infty
$$

which implies

$$
\lim _{n \rightarrow \infty} \sum_{j}\left|v_{j}^{n}-v_{j+1}^{n}\right|^{2}=0 .
$$

Using (2.2) again, we have

$$
\lim _{n \rightarrow \infty} \sum_{j}\left|u_{j}^{n}-\phi_{j}\right|^{2}=\lim _{n \rightarrow \infty} \sum_{j}\left|v_{j}^{n}-v_{j-1}^{n}\right|^{2}=0,
$$

which proves $l^{2}$ stability. Consequently, combining (2.32) and

$$
\sup _{j}\left|u_{j}^{n}-\phi_{j}\right|^{2} \leq \sum_{j}\left|u_{j}^{n}-\phi_{j}\right|^{2}
$$

we obtain

$$
\sup _{j}\left|u_{j}^{n}-\phi_{j}\right| \rightarrow 0 \text { as } n \rightarrow \infty,
$$

which proves Theorem 1.1.

Finally, we turn to the proof of Lemma 2.3.

Proof of Lemma 2.3. From (2.17) we know that

$$
(W Q)^{\prime \prime}(u)=2,
$$

so

$$
(W Q)^{\prime}\left(\phi_{j}\right)-(W Q)^{\prime}\left(\phi_{j+1}\right)=2\left(\phi_{j}-\phi_{j+1}\right) .
$$


From the equation of $\phi_{j},(1.8 \mathrm{a})$, we have

$$
\begin{aligned}
\left|\phi_{j+1}-2 \phi_{j}+\phi_{j-1}\right| & =\left|\frac{\lambda}{\alpha} f^{\prime}\left(\tilde{\phi}_{j}\right)\left(\phi_{j+1}-\phi_{j}\right)\right| \\
& =\lambda O(1)\left(\phi_{j}-\phi_{j+1}\right)
\end{aligned}
$$

and

$$
\phi_{j}-\phi_{j+1}=O(1) \lambda,
$$

which follows from (2.21). Here and in what follows we set

$$
Q_{j}=Q\left(\phi_{j}\right), \quad Q_{j}^{\prime}=Q^{\prime}\left(\phi_{j}\right)=f^{\prime}\left(\phi_{j}\right)=\Lambda_{j}
$$

without danger of confusion. Thus, we estimate $A_{j}$ as follows:

$$
\begin{aligned}
& A_{j}=\alpha\left(W_{j}-\frac{W_{j+1}+W_{j-1}}{2}\right)-\lambda\left(f_{j+1}^{\prime} W_{j+1}-f_{j}^{\prime} W_{j}\right) \\
& =\alpha\left(\frac{W_{j}-W_{j+1}}{2}+\frac{W_{j}-W_{j-1}}{2}\right)-\lambda\left(Q_{j+1}^{\prime} W_{j+1}-Q_{j}^{\prime} W_{j}\right) \\
& =\frac{W_{j}-W_{j+1}}{\phi_{j}-\phi_{j+1}} \alpha \frac{\phi_{j}-\phi_{j+1}}{2}+\frac{W_{j}-W_{j-1}}{\phi_{j}-\phi_{j-1}} \alpha \frac{\phi_{j}-\phi_{j-1}}{2}-\lambda\left(Q_{j+1}^{\prime} W_{j+1}-Q_{j}^{\prime} W_{j}\right) \\
& =-\lambda\left[\frac{W_{j}-W_{j+1}}{\phi_{j}-\phi_{j+1}} \cdot \frac{Q_{j+1}+Q_{j}}{2}-\frac{W_{j-1}-W_{j}}{\phi_{j-1}-\phi_{j}} \frac{Q_{j}+Q_{j-1}}{2}+Q_{j+1}^{\prime} W_{j+1}-Q_{j}^{\prime} W_{j}\right] \\
& =-\lambda\left[\left(W_{j+1}^{\prime}+\frac{1}{2} W_{j+1}^{\prime \prime}\left(\phi_{j}-\phi_{j+1}\right)+\frac{W^{\prime \prime \prime}\left(\xi_{j}\right)}{6}\left(\phi_{j}-\phi_{j+1}\right)^{2}\right)\right. \\
& \times\left(Q_{j+1}+\frac{Q_{j+1}^{\prime}}{2}\left(\phi_{j}-\phi_{j+1}\right)+\frac{Q^{\prime \prime}\left(\eta_{j}\right)}{4}\left(\phi_{j}-\phi_{j+1}\right)^{2}\right)+Q_{j+1}^{\prime} W_{j+1} \\
& -\left(W_{j}^{\prime}+\frac{W_{j}^{\prime \prime}}{2}\left(\phi_{j-1}-\phi_{j}\right)+\frac{W^{\prime \prime \prime}\left(\xi_{j-1}\right)}{6}\left(\phi_{j-1}-\phi_{j}\right)^{2}\right) \\
& \left.\times\left(Q_{j}+\frac{Q_{j}^{\prime}}{2}\left(\phi_{j+1}-\phi_{j}\right)+\frac{Q^{\prime \prime}\left(\eta_{j-1}\right)}{4}\left(\phi_{j-1}-\phi_{j}\right)^{2}\right)-Q_{j}^{\prime} W_{j}\right] \\
& =-\lambda\left[J_{1}+\frac{1}{2} J_{2}+\frac{1}{2} J_{3}+O(1)\left(\left(\phi_{j}-\phi_{j+1}\right)^{2}+\left(\phi_{j-1}-\phi_{j}\right)^{2}\right)\right] .
\end{aligned}
$$

Next we estimate $J_{i}(1=1,2,3)$, by using (2.35), (2.36) and (2.37), to obtain

$$
\begin{aligned}
J_{1} & =\left(W_{j+1}^{\prime} Q_{j+1}+Q_{j+1}^{\prime} W_{j+1}\right)-\left(W_{j}^{\prime} Q_{j}+Q_{j}^{\prime} W_{j}\right) \\
& =(W Q)_{j+1}^{\prime}-(W Q)_{j}^{\prime} \\
& =2\left(\phi_{j+1}-\phi_{j}\right), \\
\left|J_{2}\right| & =\left|W_{j+1}^{\prime} Q_{j+1}^{\prime}\left(\phi_{j}-\phi_{j+1}\right)-W_{j}^{\prime} Q_{j}^{\prime}\left(\phi_{j-1}-\phi_{j}\right)\right| \\
& =\left|\left(W_{j+1}^{\prime} Q_{j+1}^{\prime}-W_{j}^{\prime} Q_{j}^{\prime}\right)\left(\phi_{j}-\phi_{j+1}\right)+W_{j}^{\prime} Q_{j}^{\prime}\left(2 \phi_{j}-\phi_{j+1}-\phi_{j-1}\right)\right| \\
& =O(1)\left(\phi_{j}-\phi_{j+1}\right)^{2}+\lambda C\left(\phi_{j}-\phi_{j+1}\right) \\
& =\lambda O(1)\left(\phi_{j}-\phi_{j+1}\right) .
\end{aligned}
$$


In a similar way we have

$$
\begin{aligned}
\left|J_{3}\right| & =\left|W_{j+1}^{\prime \prime} Q_{j+1}\left(\phi_{j}-\phi_{j+1}\right)-W_{j}^{\prime \prime} Q_{j}\left(\phi_{j-1}-\phi_{j}\right)\right| \\
& =\left|\left(W_{j+1}^{\prime \prime} Q_{j+1}-W_{j}^{\prime \prime} Q_{j}\right)\left(\phi_{j}-\phi_{j+1}\right)+W_{j}^{\prime \prime} Q_{j}\left(2 \phi_{j}-\phi_{j+1}-\phi_{j-1}\right)\right| \\
& =O(1)\left(\phi_{j}-\phi_{j+1}\right)^{2}+\lambda C\left(\phi_{j}-\phi_{j+1}\right) \\
& =\lambda O(1)\left(\phi_{j}-\phi_{j+1}\right),
\end{aligned}
$$

where we have used (2.37). Substituting these into $A_{j}$, we get

$$
\begin{aligned}
A_{j} & \geq \lambda\left(\phi_{j}-\phi_{j+1}\right)\left[2-\lambda O(1)-O(1)\left(\phi_{j}-\phi_{j+1}\right)\right] \\
& \geq \lambda \nu\left(\phi_{j}-\phi_{j+1}\right),
\end{aligned}
$$

provided $\lambda$ is suitably small. Combining the above estimates proves Lemma 2.3.

\section{The $l^{1}$ STABILITY}

In this section we turn our attention to the $l^{1}$ stability of stationary discrete shocks. As is well known, $l^{1}$ is the natural norm for stability of shock waves. The main idea of achieving an $l^{1}$ estimate is to exploit the fact that the L-F scheme is essentially monotonic and to employ a carefully chosen weight function.

To obtain the $l^{1}$ estimate, (1.13), on the solution, $u_{j}^{n}$, to the L-F scheme, we will work with $v_{j}^{n}$ as in $\S 2$. We first rewrite (2.6) as

$$
\begin{aligned}
v_{j}^{n+1}- & \frac{1}{2}\left(\alpha+\lambda \Lambda_{j}\right) v_{j-1}^{n}-\frac{1}{2}\left(\alpha-\lambda \Lambda_{j+1}\right) v_{j+1}^{n} \\
& -\left(1-\alpha+\frac{\lambda}{2}\left(\Lambda_{j+1}-\Lambda_{j}\right)\right) v_{j}^{n}=e_{j}^{n} .
\end{aligned}
$$

By the CFL condition (1.5) and $\alpha<1$ we see that the coefficients of the terms $-v_{j-1}^{n}, \quad-v_{j+1}^{n}$ and $-v_{j}^{n}$ on the left side of (3.1) are all positive for small $\lambda$. This implies immediately that

$$
\begin{aligned}
& \left|v_{j}^{n+1}\right|-\frac{1}{2}\left(\alpha+\lambda \Lambda_{j}\right)\left|v_{j-1}^{n}\right|-\frac{1}{2}\left(\alpha-\lambda \Lambda_{j+1}\right)\left|v_{j+1}^{n}\right| \\
& -\left(1-\alpha+\frac{\lambda}{2}\left(\Lambda_{j+1}-\Lambda_{j}\right)\right)\left|v_{j}^{n}\right| \leq\left|e_{j}^{n}\right|,
\end{aligned}
$$

which can be rewritten as

$$
\begin{aligned}
\left|v_{j}^{n+1}\right| & -\left|v_{j}^{n}\right|+\frac{\lambda}{2} \Lambda_{j+1}\left(\left|v_{j+1}^{n}\right|-\left|v_{j}^{n}\right|\right)+\frac{\lambda}{2} \Lambda_{j}\left(\left|v_{j}^{n}\right|-\left|v_{j-1}^{n}\right|\right) \\
& -\frac{\alpha}{2}\left(\left|v_{j+1}^{n}\right|-2\left|v_{j}^{n}\right|+\left|v_{j-1}^{n}\right|\right) \leq\left|e_{j}^{n}\right| .
\end{aligned}
$$

Summing up (3.3) with respect to $j$ and using summation by parts, we obtain

$$
\sum_{j}\left|v_{j}^{n+1}\right|-\sum_{j}\left|v_{j}^{n}\right|+\lambda \sum_{j}\left(\Lambda_{j}-\Lambda_{j+1}\right)\left|v_{j}^{n}\right| \leq \sum_{j}\left|e_{j}^{n}\right|,
$$

where we have used $v_{j}^{n} \rightarrow 0$ as $j \rightarrow \pm \infty$. Since $\Lambda_{j}-\Lambda_{j+1}$ in general changes sign, owing to the nonconvexity of $f$, one cannot obtain a useful estimate. In order 
to overcome this difficulty, we multiply both sides of (3.3) by a weight function $W_{j}=W\left(\phi_{j}\right)$ to obtain

$$
\begin{gathered}
\left|v_{j}^{n+1}\right| W_{j}-\left|v_{j}^{n}\right| W_{j}+\frac{\lambda}{2} \Lambda_{j+1}\left(\left|v_{j+1}^{n}\right|-\left|v_{j}^{n}\right|\right) W_{j}+\frac{\lambda}{2} \Lambda_{j}\left(\left|v_{j}^{n}\right|-\left|v_{j-1}^{n}\right|\right) W_{j} \\
-\frac{\alpha}{2} W_{j}\left(\left|v_{j+1}^{n}\right|-2\left|v_{j}^{n}\right|+\left|v_{j-1}^{n}\right|\right) \leq W_{j}\left|e_{j}^{n}\right| .
\end{gathered}
$$

Summing up (3.5) with respect to $j$ and using summation by parts, we obtain

$$
\begin{gathered}
\sum_{j}\left|v_{j}^{n+1}\right|_{w}-\sum_{j}\left|v_{j}^{n}\right|_{w}+\frac{\lambda}{2} \sum_{j} \Lambda_{j+1}\left(\left|v_{j+1}^{n}\right|-\left|v_{j}^{n}\right|\right)\left(W_{j}+W_{j+1}\right) \\
-\frac{\alpha}{2} \sum_{j}\left|v_{j}^{n}\right|\left(W_{j+1}-2 W_{j}+W_{j-1}\right) \leq\left|e_{j}^{n}\right|_{w},
\end{gathered}
$$

that is,

$$
\sum_{j}\left|v_{j}^{n+1}\right|_{w}-\sum_{j}\left|v_{j}^{n}\right|_{w}+\sum_{j} \bar{A}_{j}\left|v_{j}^{n}\right| \leq\left|e_{j}^{n}\right|_{w},
$$

where we use the notation

$$
\begin{gathered}
\left|v_{j}^{n}\right| W_{j}=\left|v_{j}^{n}\right|_{w} \\
\bar{A}_{j}=\alpha\left(W_{j}-\frac{W_{j+1}+W_{j-1}}{2}\right)-\lambda\left(\Lambda_{j+1} \frac{W_{j+1}+W_{j}}{2}-\Lambda_{j} \frac{W_{j}+W_{j-1}}{2}\right) .
\end{gathered}
$$

The key step is to choose a suitable weight function $W$ such that $\bar{A}_{j}>0$. Comparing its form with $A_{j}$ in $\S 2$, we use the weight defined in (2.17). More precisely, we have the following lemma.

Lemma 3.1. Let $W_{j}$ be the weight defined in (2.17). Then we can choose $\lambda$ suitably small so that

$$
\bar{A}_{j} \geq \nu_{1} \lambda\left(\phi_{j}-\phi_{j+1}\right)
$$

Assuming this lemma for a moment, we have from (3.7) and (3.10) that

$$
\sum_{j}\left|v_{j}^{n+1}\right|_{w}-\sum_{j}\left|v_{j}^{n}\right|_{w}+\lambda \sum_{j}\left(\phi_{j}-\phi_{j+1}\right)\left|v_{j}^{n}\right| \leq O(1)\left|e_{j}^{n}\right|_{w} .
$$

A simple calculation, using the definition of $e_{j}^{n}$, shows that

$$
\begin{aligned}
\left|e_{j}^{n}\right|_{w} & \leq \frac{\lambda}{2}\left(\left|\theta_{j+1}^{n}\right|_{w}+\left|\theta_{j-1}^{n}\right|_{w}\right) \\
& \leq O(1)\left(\left|v_{j+1}^{n}-v_{j}^{n}\right|_{w}^{2}+\left|v_{j}^{n}-v_{j-1}^{n}\right|_{w}^{2}\right),
\end{aligned}
$$

which, together with (3.11), implies that, for any $n_{2} \in Z^{+}$,

$$
\begin{gathered}
\sum_{j}\left|v_{j}^{n_{2}+1}\right|_{w}-\sum_{j}\left|v_{j}^{0}\right|_{w}+\lambda \sum_{n=0}^{n_{2}} \sum_{j}\left(\phi_{j}-\phi_{j+1}\right)\left|v_{j}^{n}\right| \\
\leq O(1) \sum_{n=0}^{n_{2}}\left|v_{j+1}^{n}-v_{j}^{n}\right|_{w}^{2} .
\end{gathered}
$$

But Proposition 2.2 shows that the last term on the right-hand side of (3.12) is bounded from above by $O(1) N^{2}(0)$. On the other hand, our weights are bounded from both above and below by some positive constants. Thus, we have shown: 
Proposition 3.1. Assume that $N(0)$ is suitably small. Then the problem (3.1) has a unique global solution $v_{j}^{n}$ satisfying, for any $n \geq 0$,

$$
\sup _{n} \sum_{j}\left|v_{j}^{n}\right|+\sum_{j, n}\left(\phi_{j}-\phi_{j+1}\right)\left|v_{j}^{n}\right| \leq C \sum_{j}\left(\left|v_{j}^{0}\right|+\left|v_{j}^{0}\right|^{2}\right) .
$$

With this proposition, we can prove Theorem 1.2 as follows.

Proof of Theorem 1.2. First we prove in a similar way as in the proof of Theorem 1.1 that the condition (1.12) in Theorem 1.2 on the initial data implies that $N(0)$ is small. Thus, the hypothesis in Proposition 3.1 is fulfilled under the condition (1.12), which implies the global existence of a solution, $u_{j}^{n}$, to the L-F scheme (1.4) because of the relation

$$
u_{j}^{n}=\phi_{j}+v_{j}^{n}-v_{j-1}^{n},
$$

which follows from (2.2). From Proposition 3.1 and (2.2), we have

$$
\sum_{j}\left|u_{j}^{n}-\phi_{j}\right| \leq 2 \sum_{j}\left|v_{j}^{n}\right|<\infty,
$$

which yields the desired estimate (1.13).

Finally, we turn to the proof of Lemma 3.1.

Proof of Lemma 3.1. Using the weight function $W_{j}$ defined in (2.17), by virtue of (2.36) and (2.37), we estimate $\bar{A}_{j}$ as follows:

$$
\begin{aligned}
\bar{A}_{j}= & \alpha\left(W_{j}-\frac{W_{j+1}+W_{j-1}}{2}\right)-\lambda\left(\Lambda_{j} \frac{W_{j+1}+W_{j}}{2}-\Lambda_{j} \frac{W_{j}+W_{j-1}}{2}\right) \\
= & \alpha\left(W_{j}-\frac{W_{j+1}+W_{j-1}}{2}\right) \\
& -\lambda\left[\Lambda_{j}\left(W_{j+1}+W_{j+1}^{\prime} \frac{\phi_{j}-\phi_{j+1}}{2}+\frac{W^{\prime \prime}\left(\xi_{j+1}\right)}{4}\left(\phi_{j}-\phi_{j+1}\right)^{2}\right)\right. \\
& \left.\quad-\Lambda_{j}\left(W_{j}+W_{j}^{\prime} \frac{\phi_{j}-\phi_{j-1}}{2}+\frac{W^{\prime \prime}\left(\xi_{j}\right)}{4}\left(\phi_{j-1}-\phi_{j}\right)^{2}\right)\right] \\
= & \alpha\left(W_{j}-\frac{W_{j+1}+W_{j-1}}{2}\right)-\lambda\left(\Lambda_{j} W_{j+1}-\Lambda_{j} W_{j}\right) \\
& -\frac{\lambda}{2}\left[\Lambda_{j} W_{j+1}^{\prime}\left(\phi_{j}-\phi_{j+1}\right)+\Lambda_{j} W_{j}^{\prime}\left(\phi_{j}-\phi_{j-1}\right)\right]+O(1)\left(\phi_{j}-\phi_{j+1}\right)^{2} \\
= & A_{j}-\frac{\lambda}{2}\left[\left(\Lambda_{j} W_{j+1}^{\prime}-\Lambda_{j} W_{j}^{\prime}\right)\left(\phi_{j}-\phi_{j+1}\right)\right. \\
& \left.+\Lambda_{j} W_{j}^{\prime}\left(2 \phi_{j}-\phi_{j+1}-\phi_{j-1}\right)\right]+O(1)\left(\phi_{j}-\phi_{j+1}\right)^{2} \\
= & A_{j}-O(1) \lambda\left(\phi_{j}-\phi_{j+1}\right) .
\end{aligned}
$$

With the weight function defined in (2.17) we have Lemma 2.1, which yields

$$
\begin{aligned}
\bar{A}_{j} & \geq \lambda(\nu-O(1) \lambda)\left(\phi_{j}-\phi_{j+1}\right) \\
& \geq \lambda \nu_{1}\left(\phi_{j}-\phi_{j+1}\right)
\end{aligned}
$$

for a positive constant $\nu_{1}$, provided $\lambda$ is suitably small. 


\section{REFERENCES}

1. G.Q. Chen, Convergence of the Lax-Friedrichs schemes for isentropic gas dynamics(III), Acta Mathematica Scientia, 6(1986), 75-120. MR 89f:76007

2. I. L. Chern, Large-time behavior of solutions of Lax-Friedrichs Finite Difference Equations for hyperbolic systems of conservation laws, Math Comp., 56(1991),107-118. MR 91j:65148

3. X.X. Ding, G.Q. Chen and P.Z. Luo, Convergence of the Lax-Friedrichs schemes for isentropic gas dynamics(I), Acta Mathematica Scientia, 5(1985), 415-432. MR 89f: 76006

4. X.X. Ding, G.Q. Chen and P. Z.Luo, Convergence of the Lax-Friedrichs schemes for isentropic gas dynamics(II), Acta Mathematica Scientia, 5(1985), 433-472. MR 89f:76006

5. R.J. DiPerna, Convergence of approximate solutions to conservation laws, Arch. Rat. Mech. Anal., 82(1983), 27-70. MR 84k:35091

6. B. Engquist and S. Osher, One-sided difference approximations for nonlinear conservation laws, Math. Comp., 36(1981), 321-351. MR 82c:65056

7. J. Goodman, Nonlinear asymptotic stability of viscous shock profiles to conservation laws, Arch. Rat. Mech. Anal. 95(1986), 325-344. MR 88b:35127

8. E. Harabetian, Rarefaction and large-time behavior for parabolic equations and monotone schemes, Comm. Math. Phys. 114(1988), 527-536. MR 89d:35084

9. G. Jennings, Discrete shocks, Comm.Pure Appl. Math. 27(1974),25-37. MR 49:3358

10. C.K.R.T. Jones, R. Gardner and T. Kapitula, Stability of travelling waves for nonconvex scalar viscous conservation laws, Comm. Pure Appl. Math., 46(1993)505-526. MR 94c:35123

11. S. Kawashima and A. Matsumura, Asymptotic stability of travelling wave solutions of systems for one-dimensional gas motion, Comm. Math. Phys., 101(1985), 97-127. MR 87h:35035

12. S. Kawashima and A. Matsumura, Stability of shock profiles in viscoelasticity with nonconvex constitutive relations, preprint(1993).

13. N. N. Kuznecov and S.A. Vološin, On monotone difference approximations for a first-order quasi-linear equation, Soviet Math. Dokl. 17(1976),1203-1206. MR 54:4126

14. P.D. Lax, Weak solutions of nonlinear hyperbolic equations and their numerical computation, Comm. Pure Appl. Math. 7(1954), 159-193. MR 16:524g

15. P. D. Lax and B. Wendroff, Systems of conservation laws, Comm. Pure Appl. Math., 13(1960), 217-237. MR 22:11523

16. H. L. Liu, Asymptotic stability of shock waves for nonconvex Convection-Diffusion equation, preprint(1994).

17. T. P. Liu, Nonlinear stability of shock waves for viscous conservation laws, Mem. Amer. Math. Soc., 56, no.328(1985). MR 87a:35127

18. J. G. Liu and Z. P. Xin, Nonlinear stability of discrete shocks for systems of conservation laws, Arch. Rat. Mech. Anal., 125(1993), 217-256. MR 95c:35166

19. G. Liu and Z. P. Xin, $L^{1}$ stability of stationary discrete shocks, Math. Comp. 60(1993), 233-244. MR 93d:35097

20. A. Majda and J. Ralston, Discrete shock profiles for systems of conservation laws, Comm. Pure Appl. Math. 43(1979), 445-483. MR 81i:35108

21. H. Nessyahu and E. Tadmor, Non-oscillatory central differencing for hyperbolic conservation laws, J. Comp. Phys. 87(1990), 408-463. MR 91i:65157

22. O. Oleinik, Discontinuous solutions of nonlinear differential equations, Usp. Mat. Nauk., 27(1957), 3-73. MR 20:1055

23. R. Sanders, On convergence of monotone finite difference schemes with variable spatial differencing, Math. Comp. 40(1983), 91-106. MR 84a:65075

24. D. Serre, $L^{1}$ stability of viscous shock waves for scalar nonconvex conservation law, (Private Communication)(1994)

25. Y.S. Smyrlis, Existence and Stability of stationary profiles of the LW scheme. Comm. Pure Appl. Math., 43(1990), 508-545. MR 91d:65143

26. A. Szepessy and Z. Xin, Nonlinear stability of viscous shock waves, Arch. Rat. Mech.Anal. 122(1993) 53-104. MR 93m:35125

27. E. Tadmor, The large-time behavior of the scalar genuinely nonlinear Lax-Friedrichs scheme, Math. Comp. 43(1984), 353-368. MR 86g:65162

28. Z.H. Teng and P.W. Zhang, Optimal $L^{1}$-Rate of convergence for viscosity method and monotone scheme to piecewise constant solutions with shocks, Institute of Mathematics and Department of Mathematics, Peking University, Research Report No.28 (1994). 
29. L.A. Ying and T. Zhou, Long-time asymptotic behavior of Lax-Friedrichs Scheme, J. Partial Diff. Eqs., 6(1)(1993), 39-61. MR 94b:35173

Department of Mathematics, Henan Normal University, XinXiang 453002, P. R. China

E-mail address: guozm@sun.ihep.ac.cn

Institute of Systems Science, Academia Sinica, Beijing 100080, P.R.China

E-mail address: jwang@iss06.iss.ac.cn 